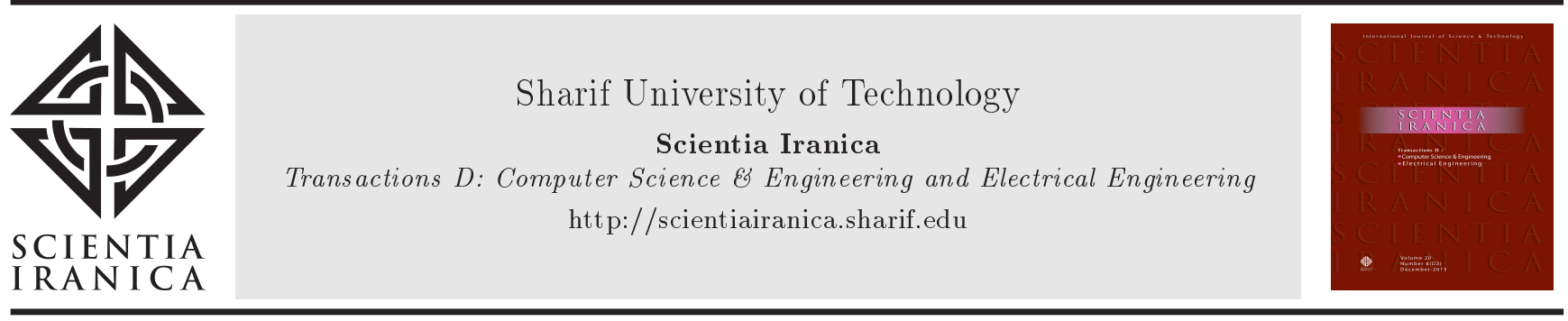

\title{
Infection detection in cystic fibrosis patients based on tunable Q-factor wavelet transform of respiratory sound signal and ensemble decision
}

\author{
A. Karimizadeh ${ }^{\mathrm{a}}$, M. Vali ${ }^{\mathrm{a}, *}$, and M.R. Modaresi $\mathbf{b}^{\mathrm{b}}$ \\ a. Department of Biomedical Engineering, K.N. Toosi University of Technology, Tehran, P.O. Box 16315-1355, Iran. \\ b. Pediatric Respiratory and Sleep Medicine Research Center, Children's Medical Center, Tehran University of Medical Sciences, \\ Tehran, Iran.
}

Received 19 February 2020; received in revised form 9 August 2020; accepted 7 December 2020

\section{KEYWORDS \\ Cystic fibrosis; \\ Respiratory sound; \\ Tunable Q-factor \\ wavelet transform; \\ Support vector \\ machine; \\ Ensemble classifier.}

\begin{abstract}
Most adult Cystic Fibrosis (CF) patients frequently suffer from Pseudomonas aeruginosa (PA) infection, which is strongly associated with inflammation, lung destruction, and increased mortality. Diagnosis of PA infection in the primary stage is essential to initiate the treatment and reduce the risk of chronic infection. Sputum culture is the gold standard for infection detection, but it is time consuming. The objective of this study was to suggest and examine a method to determine PA infection status in CF patients based only on their respiratory sound. Respiratory sounds were recorded from 36 CF patients. Some features which were generated from Tunable Q-factor Wavelet Transform (TQWT) components were investigated. The features were fed into Support Vector Machine and Ensemble classifier. The proposed method achieved an accuracy rate of $90.3 \%$ in identifying PA infection in CF patients. Furthermore, the probability of categorizing respiratory sounds as PA CF decreased significantly after the treatment of PA infection $(P$-value $<$ 0.003). Moreover, the method exhibited a satisfactory performance in the presence of noises and artifacts. The developed method represents a novel approach to the diagnosis of PA infection in CF patients based only on respiratory sound signals, which is a necessary and innovative approach to early diagnosis of PA infection.
\end{abstract}

(C) 2022 Sharif University of Technology. All rights reserved.

\section{Introduction}

Cystic Fibrosis (CF) is the most common autosomal recessive disorder in white skinned individuals that affects such organs as the lungs and pancreas. Chronic lung infections, the hallmark of $\mathrm{CF}$, are the principal cause of morbidity and mortality in CF patients. Thick

*. Corresponding author. Tel.: +9821 8406-2405 E-mail addresses: a.karimizadeh@ee.kntu.ac.ir (A. Karimizadeh); mansour.vali@eetd.kntu.ac.ir (M.Vali); mr-modaresi@sina.tums.ac.ir (M.R. Modaresi)

doi: $10.24200 /$ sci.2020.55468.4242 and sticky lung mucus is a common respiratory sign of $\mathrm{CF}$. Infections of the lungs result in pulmonary symptoms and pulmonary exacerbation in $\mathrm{CF}[1,2]$. Infections are the key parameters considered by physicians to initiate appropriate preventive and therapeutic strategies [1].

Approximately $60-75 \%$ of adult CF patients frequently suffer from Pseudomonas Aeruginosa (PA) infections [1]. The PA infection is strongly associated with inflammation, lung destruction [2], and increased risk of mortality [3-5]. Early detection of PA is essential to initiation of appropriate therapy and reduction of the risk of chronic infection [6]. During the early stages, PA can be treated using inhaled or oral 
antibiotics (instead of intravenous antibiotics). Nevertheless, the treatment procedure must be monitored to assess its effectiveness. To diagnose infections and evaluate the treatment efficiency, airway cell culture is generally recommended once every 3 months in a stable state and with the occurrence of acute pulmonary exacerbation [7]. Although the sputum culture result is the gold standard for the detection of infection, it is time consuming; hence, a real-time diagnostic method can facilitate early initiation of eradication therapy.

Evaluation of the lung sounds, which are produced during the transition of air through the respiratory tract, is a non-invasive method for investigating lung status [8]. Pulmonary diseases can alter the airways and cause variations in respiratory sounds. Identifying and categorizing information about the time and frequency of lung sounds for the diagnosis of disease and evaluation during disease follow-up are challenging without the use of computerized analysis. Recently, computerized respiratory sound analysis has been reported as a beneficial diagnostic tool to identify lung abnormalities and disorders [9-14].

Many CF patients experience exacerbation in the presence of PA infection. Decreased lung function, increased cough, increased sputum, and increased adventitial lung sounds are indicators of exacerbation. Even though there is evidence that infection can alter the airways by increasing mucus and sputum, few studies have investigated whether respiratory sounds are affected by infections [15-17]. Previous studies have only focused on the relations between adventitious lung sounds such as wheeze, crackles, or cough sounds, and respiratory infections $[15,17]$. However, a study has investigated lung sounds originating from lungs infected by Tuberculosis to discriminate healthy subjects from Tuberculosis patients [16].

There are few studies that investigated relations between adventitious lung sounds and infection. In [15], cough sounds were analyzed to identify respiratory infection in pigs. In another study, wheeze lung sounds were detected to diagnose and monitor lower respiratory tract infection [17]. The role of respiratory physiotherapy was examined in lower respiratory tract infections in another study, which caused a significant reduction in wheeze rates and less sputum after respiratory physiotherapy [18]. In the literature, there are several uses of respiratory sound frequency analysis [19,20] and time-frequency analysis (especially Wavelet Transform) in the automatic diagnosis of adventitious respiratory sounds [8].

Rational-Dilation Wavelet Transform (RADWT) [21] and Tunable Q-factor Wavelet Transform (TQWT) [22] are kinds of wavelet transform that describe a signal as an optimum time-frequency representation by choosing an appropriate Q-factor [22]. It was shown in [23] that these features resulted in better accuracy than discrete wavelet transform, PSD, MFCC, and some other features in the diagnosis of adventitious respiratory sounds. In RADWT, the Q-factor is adjusted using the combined action of three parameters, while in TQWT, it is specified directly [24]. Recently, high Q-factor and low Q-factor features have been used in the diagnosis of adventitious respiratory sounds [23]. Altogether, these studies on the diagnosis of adventitious respiratory sounds, though not directly relevant to this study, may lead us to the hypothesis that the features extracted from the mentioned techniques may be capable of diagnosing a sound beyond the human auditory judgment, which is consistent with infection in $\mathrm{CF}$ patients.

Structural airway alterations may modify lung sounds by increasing mucus and sputum which can be used to discriminate Normal CF from PA CF patients' lung sounds. Recent studies have demonstrated that respiratory sounds are affected by sputum conditions [25-27]. Four distinct research works have been carried out to examine the sputum condition using respiratory sounds. The first research utilized a frequency domain feature to examine the effect of increased sputum on respiratory sounds [28]. For this purpose, sound samples of three patients were used to detect increase in sputum with $85-97 \%$ accuracy. Two other works examined time-frequency features [25] and discrete wavelet transform features [27] to diagnose sputum accumulation in the trachea. Using sound samples of 12 patients, they achieved accuracy rates of $83.5 \%$ and $84.5 \%$ employing time-frequency features and discrete wavelet transform features, respectively. Recently, another work has utilized sound samples of 14 patients to evaluate features based upon Empirical Mode Decomposition (EMD) and achieved an accuracy of $92.02 \%$ in the diagnosis of sputum condition [26]. All of these studies, however, depicted the effectiveness of time-frequency features and features from basic oscillatory portions of signal (EMD of the signal) to diagnose sputum conditions.

Mucus clusters vibrate due to air transition produced by respiration. It was mentioned in [25] that the vibration in time-frequency representation of respiratory sound signals might be created by small oscillations in the signal. Therefore, TQWT analysis can be used to obtain optimal resonance-based respiratory sound signal decomposition to detect these oscillations.

This study was done in the CF center of the children hospital; thus, the participants were only $\mathrm{CF}$ patients. The diagnosis of $\mathrm{CF}$ for the participants were confirmed based on sweet test and/or genetic test. The gold standard method for the detection of PA infection in CF patients is time consuming (several days); hence, a real-time diagnostic method may facilitate early initiation of eradication therapy. So far, there has been no attempt to detect PA infection 
in CF patients using respiratory sounds. The proposed study is, therefore, an attempt to address this issue with the aim of detecting PA infection in CF patient by analyzing only one cycle of respiratory sound instead of detecting adventitious lung sounds. For this purpose, a number of features extracted from TQWT components of respiratory sounds were evaluated in identifying PA infection in $\mathrm{CF}$ patients.

\section{Material and methods}

The overall structure of the proposed method is illustrated in Figure 1. After describing data acquisition part, each step in Figure 1 is described in detail.

\subsection{Data acquisition}

Respiratory sounds were acquired from $25 \mathrm{CF}$ patients (11 females, 14 males) whom were followed up at the Pediatric Respiratory and Sleep Medicine Research Center of Children's Medical Center. The diagnosis of $\mathrm{CF}$ for the participants was confirmed based on sweet test and/or genetic test. Patients were selected based on their sputum microbiology cultures according to the following category: 11 patients with normal flora culture results and 14 patients with PA infection (in this paper, 'Normal CF subjects' and 'PA CF subjects' will be used to refer to normal flora CF subjects and CF subjects with PA infection, respectively). Also, respiratory sounds of 11 patients were recorded one month after antibiotic treatment and were used to investigate the effectiveness of the proposed method. Demographic data of the patients are depicted in Table 1. All procedures performed in studies involving human participants were approved (IR.TUMS.CHMC.REC.1398.094) by the Ethics Committee of Children's Medical Center-Tehran University of Medical Sciences.

Respiratory sounds were recorded with a Littman 3200 digital stethoscope in the extended mode from 20 to $2000 \mathrm{~Hz}$ with a sampling frequency of $4000 \mathrm{~Hz}$. Sound acquisition was performed from two anatomical regions of the lungs; posterior upper right and

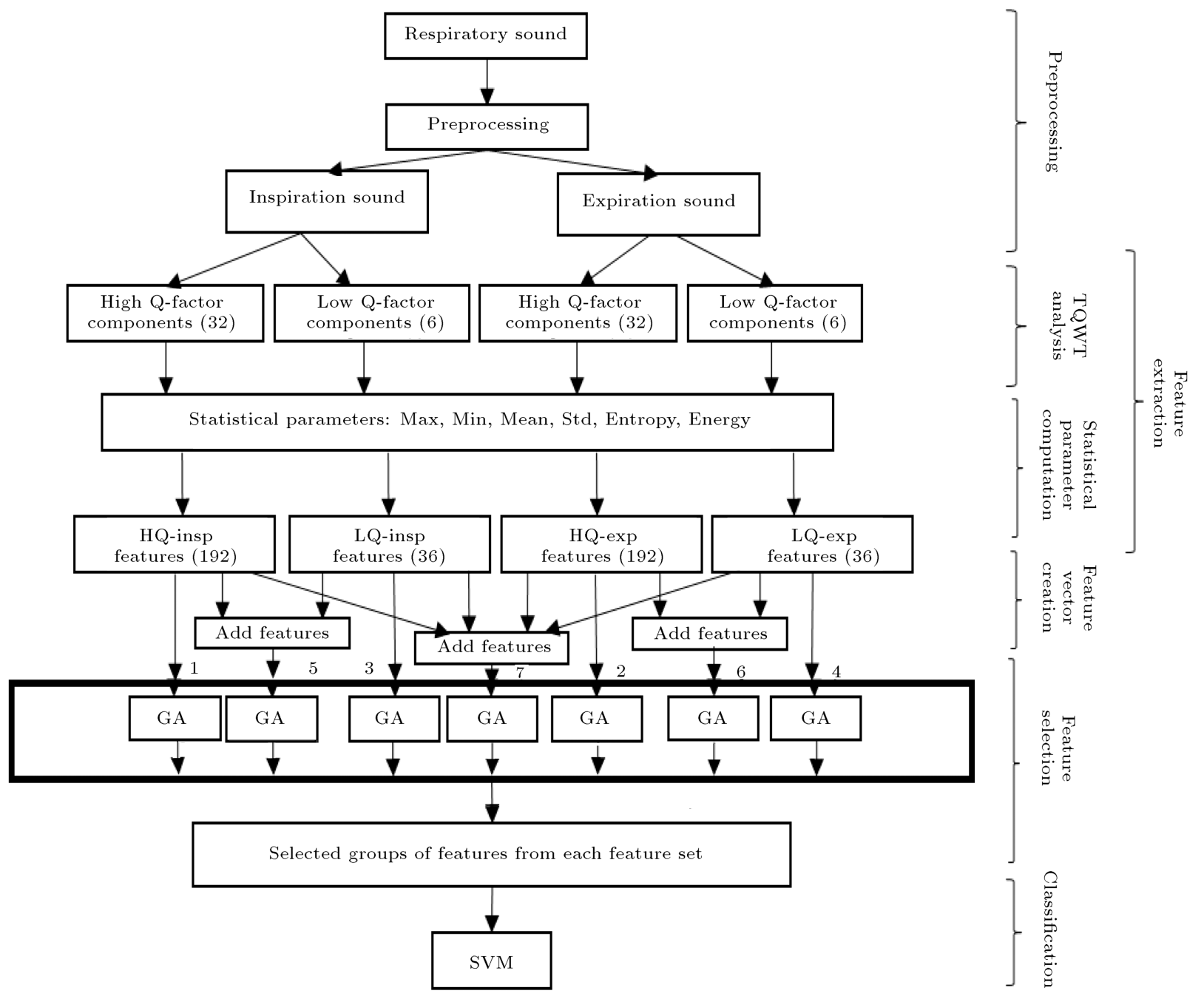

Figure 1. The overall structure of the proposed method. 
Table 1. CF participant's information.

\begin{tabular}{lccc}
\hline & $\begin{array}{c}\text { Normal CF } \\
(\boldsymbol{n}=\mathbf{1 1})\end{array}$ & $\begin{array}{c}\text { PA CF } \\
(\boldsymbol{n}=\mathbf{1 4})\end{array}$ & $\begin{array}{c}\text { PA CF after treatment } \\
(\boldsymbol{n}=\mathbf{1 1})\end{array}$ \\
\hline Gender (female/male) & $5 / 6$ & $6 / 8$ & $5 / 6$ \\
Age (mean \pm std) & $12.18 \pm 3.9$ & $15.1 \pm 5.3$ & $15.5 \pm 5.7$ \\
Height (mean \pm std) & $147.2 \pm 20.1$ & $149.5 \pm 17.4$ & $153.7 \pm 12.3$ \\
Weight (mean \pm std) & $36.1 \pm 12.1$ & $37.3 \pm 11.6$ & $40.4 \pm 12.5$ \\
\hline
\end{tabular}

left. Over two normal respiratory sound cycles were recorded from each volunteer in the sitting position. Informed consent was obtained from all patients or their parents in case of under ages prior to respiratory sound recording.

\subsection{Preprocessing}

As shown in Figure 1, the first step is preprocessing, which includes noise and artifact removal and respiratory cycle separation. Recorded sounds were contaminated with the cardiac sound artifact, speaking and coughing noises, and digital stethoscope movement noise. The dominant frequency of heart sound is lower than $150 \mathrm{~Hz}$ [29]. To remove the cardiac sound effect, band-pass filtering was applied to the recorded respiratory sounds in the frequency range of $150-1800$ Hz. Respiratory sound cycles damaged by speaking or coughing noises were excluded from the dataset. Digital stethoscope movement noises were high amplitude spikes with very low lengths, which were removed from the signals based on the method in [30].

The recorded signals had good quality. Three reviewers listened to the recorded sounds, independently. All respiratory sound samples were listened precisely. Their spectrograms were investigated as well. Samples that contained speaking noises and special lines related to the speaking in their spectrogram were omitted from the study. From 157 available respiratory sound samples, 21 samples were contaminated with noises and omitted from the study. Finally, 136 remaining sound samples were used for the study. Totally, 47, 67, and 22 respiratory sound cycles were achieved from normal CF, PA CF patients and PA CF patients after the treatment, respectively. Then, inspiration and expiration sound segments were separated from respiratory sound cycles manually.

The proposed method used preprocessed respiratory sound signals. However, noisy respiratory sound signals were utilized to evaluate the functionality of the proposed method in the presence of noises and artifacts.

\subsection{Feature extraction}

The proposed feature extraction method includes the decomposition of respiratory sound signals into their TQWT components and the calculation of statistical parameters from the obtained components (Figure 1). In TQWT analysis, a two-channel filter bank is applied to a signal and then, the scaled version of the filter bank is iteratively applied to its low-pass parts. It is assumed that $\alpha$ and $\beta$ are scaling parameters of lowpass $H_{0}(\boldsymbol{\omega})$ and high-pass $H_{1}(\boldsymbol{\omega})$ filters, respectively, and $0 \leq j \leq J$ is the index of decomposition level for a $J$ level TQWT transform.

$$
\begin{array}{ll}
H_{0}(\omega)=1 & |\omega| \leq(1-\beta) \pi, \\
H_{0}(\omega)=0 & \alpha \pi \leq|\omega| \leq \pi \\
H_{1}(\omega)=0 & |\omega| \leq(1-\beta) \pi, \\
H_{1}(\omega)=1 & \alpha \pi \leq|\omega| \leq \pi .
\end{array}
$$

By analyzing the cascade of several filters and scalings, the equivalent frequency response for $\alpha \leq 1$ and $\beta \leq 1$ at level $j$ of decomposition can be calculated as $H_{1}^{(j)}(\omega)$ from Eq. (5) [22]:

$$
\begin{aligned}
& H_{1}^{(j)}(\omega)= \\
& \begin{cases}H_{1}\left(\frac{\omega}{\alpha^{j-1}}\right) & \prod_{m=0}^{j-2} H_{0}\left(\frac{\omega}{\alpha^{m}}\right) \\
& (1-\beta) \alpha^{j-1} \pi \leq|\omega| \leq \alpha^{j-1} \pi \\
0 & \text { for other } \omega[-\pi, \pi]\end{cases}
\end{aligned}
$$

where $H_{0}^{(j)}(\omega)$ is calculated using Eq. (6):

$$
H_{0}^{(j)}(\omega)= \begin{cases}\prod_{m=0}^{j-1} H_{0}\left(\frac{\omega}{\alpha^{m}}\right), & |\omega| \leq \alpha^{j} \pi \\ 0 & \alpha^{j} \pi<|\omega| \leq \pi\end{cases}
$$

The major parameters in TQWT include Q-factor $(Q)$, redundancy $(r)$, and number of decomposition levels $(J)$.

Q-factor is the ratio of center frequency to bandwidth of a band-pass filter. It is a measure of number of oscillations in wavelet. Eq. (7) reveals the relation between $Q$ and $\beta$ parameters:

$$
Q=\frac{\omega_{c}}{B W}=\frac{2-\beta}{\beta} .
$$

Redundancy is the total number of wavelet coefficients divided by the length of the signal. The relation between $\mathrm{r}$ and filter parameters $\alpha$ and $\beta$ is revealed in Eq. (8).

$$
r=\frac{\beta}{1-\alpha} \text {. }
$$


A signal can be decomposed into a collection of high Q-factor and/or low Q-factor components; the number of components is $J+1$.

The presence of mucus in the lung and airways due to PA infection can alter the airway structure in CF patients, subsequently causing changes in air transition and lung sounds. If any mucus is stored in a patient's airway, mucus clusters may vibrate due to air transition produced by respiration. These vibrations may cause small oscillations in special parts of respiratory sound, as mentioned in [25]. Visual evaluation of recorded respiratory sounds showed that some, but not all, of the PA infected lungs had these signs.

Therefore, TQWT analysis was used to obtain an appropriate resonance-based respiratory sound signal decomposition by selecting appropriate Q-factor to detect changes related to PA infection in CF patients. Hence, differences between components of respiratory sounds (obtained from TQWT analysis) were investigated in normal CF and PA CF patients. In this study, both high and low Q-factor components of respiratory sounds were evaluated in the detection of PA infection. However, it can be expected that high Q-factor components of respiratory sounds have better performance than low Q-factor components in distinguishing oscillations related to infection in respiratory sounds as a result of better frequency selectivity of high Q-factor wavelet bases. The redundancy factor $r$ controls overlapping rate between sub-band frequency responses of adjacent wavelets in the TQWT method. It is often recommended to be equal to or greater than 3 in order to well localize the analysis/synthesis functions (wavelets) [24,31]. Frequency responses of adjacent wavelets were investigated using different $r$ values. The $r$ was set to 3 based on some trials and for keeping a kind of balance in overlapping between wavelets of successive frequency bands. Q-factor describes the degree of resonance in a signal and affects the oscillatory behavior of the wavelet, meaning that it is a measure of the number of oscillations exhibited by the wavelet. Therefore, Qfactor, which describes the degree of resonance in a signal, should be selected according to the signal type. In this study, Q-factors were selected around the values used in the previous study on adventitious respiratory sounds using high Q-factor and low Q-factor around 6 and around 2 [23]. Then, high and low Q-factor values were obtained around these values through trial and error. Parameter $J$ was selected based on respiratory sound signal energy distribution in different subbands. $J$ was selected by increasing the level of decomposition until energy of the last subband was approximately less than $1 \%$ of the total signal energy.

Decomposition of respiratory sound cycles into high Q-factor components was done by parameters of $Q=8, r=3$, and $J=40$ and for low Q-factor components by parameters of $Q=1, r=3$, and $J=9$.
Energy distributions of 41 high Q-factor components of respiratory sounds in two groups of normal $\mathrm{CF}$ and PA CF subjects are shown in Figure 2. They are also shown for 10 low Q-factor components in Figure 3. As can be seen, there are some differences between energy distributions of high and low Q-factor components of normal CF and PA CF subjects' respiratory sounds in inspiration/expiration.

After decomposing respiratory sound signals into high and low Q-factor components, some statistical parameters were considered in order to create feature vectors from the extracted components and feed them to classifiers (Figure 1).

These parameters were maximum, minimum, mean, standard deviation, entropy, and energy which were calculated using Eqs. (9)-(14):

$$
\begin{aligned}
& \max \left(M x_{i}\right)=\max \left(S_{k i}\right) \quad \text { for } k=1, \ldots, N_{i}, \\
& \min \left(M n_{i}\right)=\min \left(S_{k i}\right) \quad \text { for } k=1, \ldots, N_{i}, \\
& \operatorname{Mean}\left(\mu_{i}\right)=\frac{\sum_{k=1}^{N} S_{k i}}{N_{i}}, \\
& \operatorname{Std}\left(\sigma_{i}\right)=\sqrt{\frac{\sum_{k=1}^{N_{i}}\left(S_{k i}-\mu_{i}\right)^{2}}{N_{i}}}, \\
& \operatorname{Entropy}\left(E_{n} t_{i}=-\sum_{k=1}^{N_{i}} S_{k i} \log \left(S_{k i}\right),\right. \\
& \text { Energy }\left(E_{i}\right)=\frac{1}{N_{i}} \sum_{k=1}^{N_{i}}\left|S_{k i}\right|^{2},
\end{aligned}
$$

where ' $S_{k i}$ ' is the $i$ th Q-factor component with the length of $N_{i}$ and $k$ is the index of samples in each component.

High Q-factor components no. 34-41 and low Q-factor components no. 7-10 contained negligible information after filtering respiratory signals in the preprocessing step. Therefore, statistical parameters were calculated from high Q-factor components no. 233 (32 components) and low Q-factor components no. 1-6 (6 components).

In this step, 32 high Q-factor components were obtained from inspiration/expiration sounds. One feature vector was created as $F V_{q}(q=1-32)$ for high Q-factor components of inspiration sound, where $q$ is the number of components.

$$
F V_{q}=\left[\begin{array}{c}
M x_{q} \\
M n_{q} \\
\mu_{q} \\
\sigma_{q} \\
E n t_{q} \\
E n_{q}
\end{array}\right]
$$

In the same way, $F V_{q}$ was created for low Q-factor 

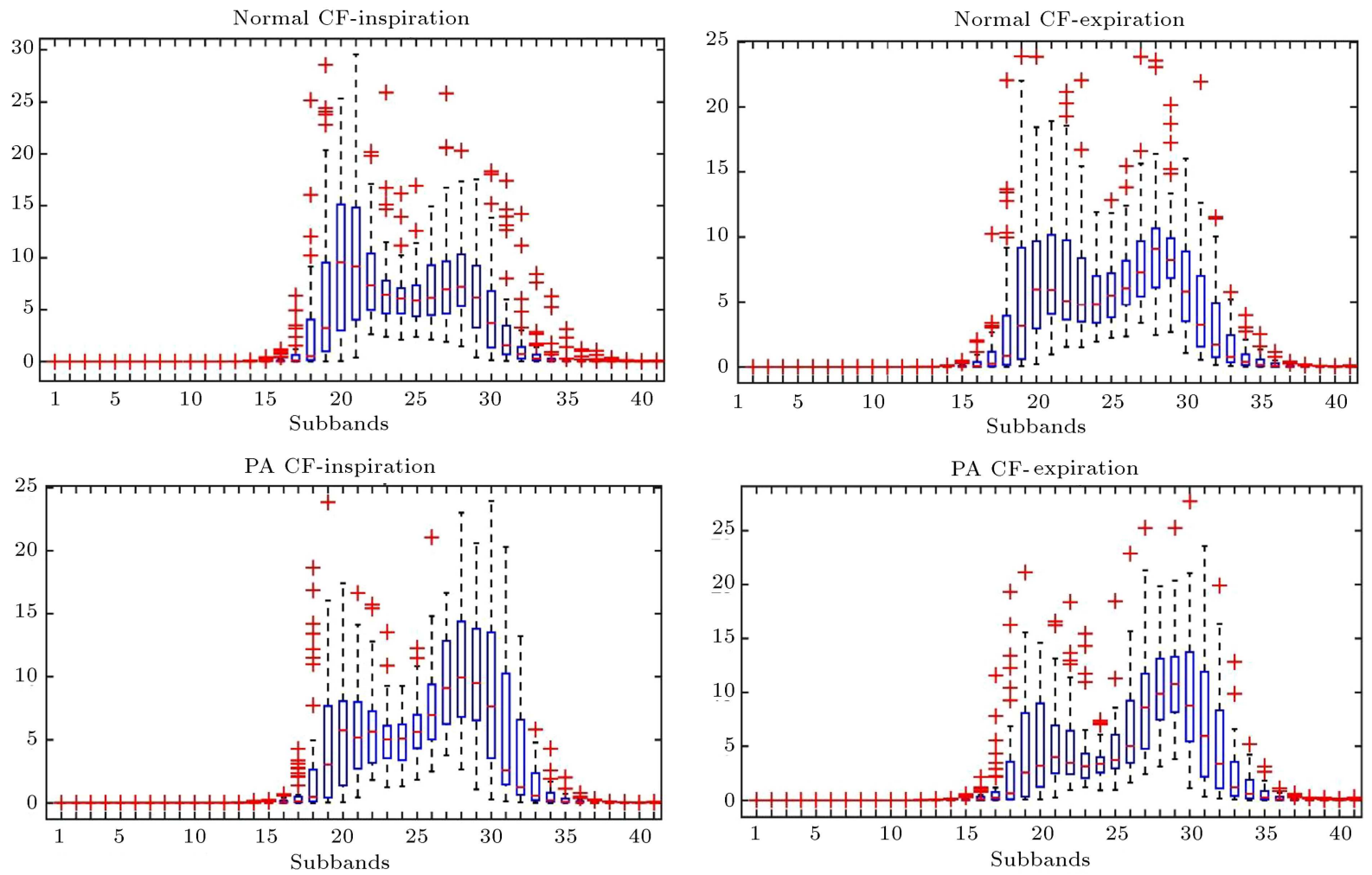

Figure 2. Energy distribution of 41 high Q-factor components of inspiration and expiration sounds in two groups of normal CF and PA CF subjects with median and InterQuartile Range (IQR) calculated across all patients.
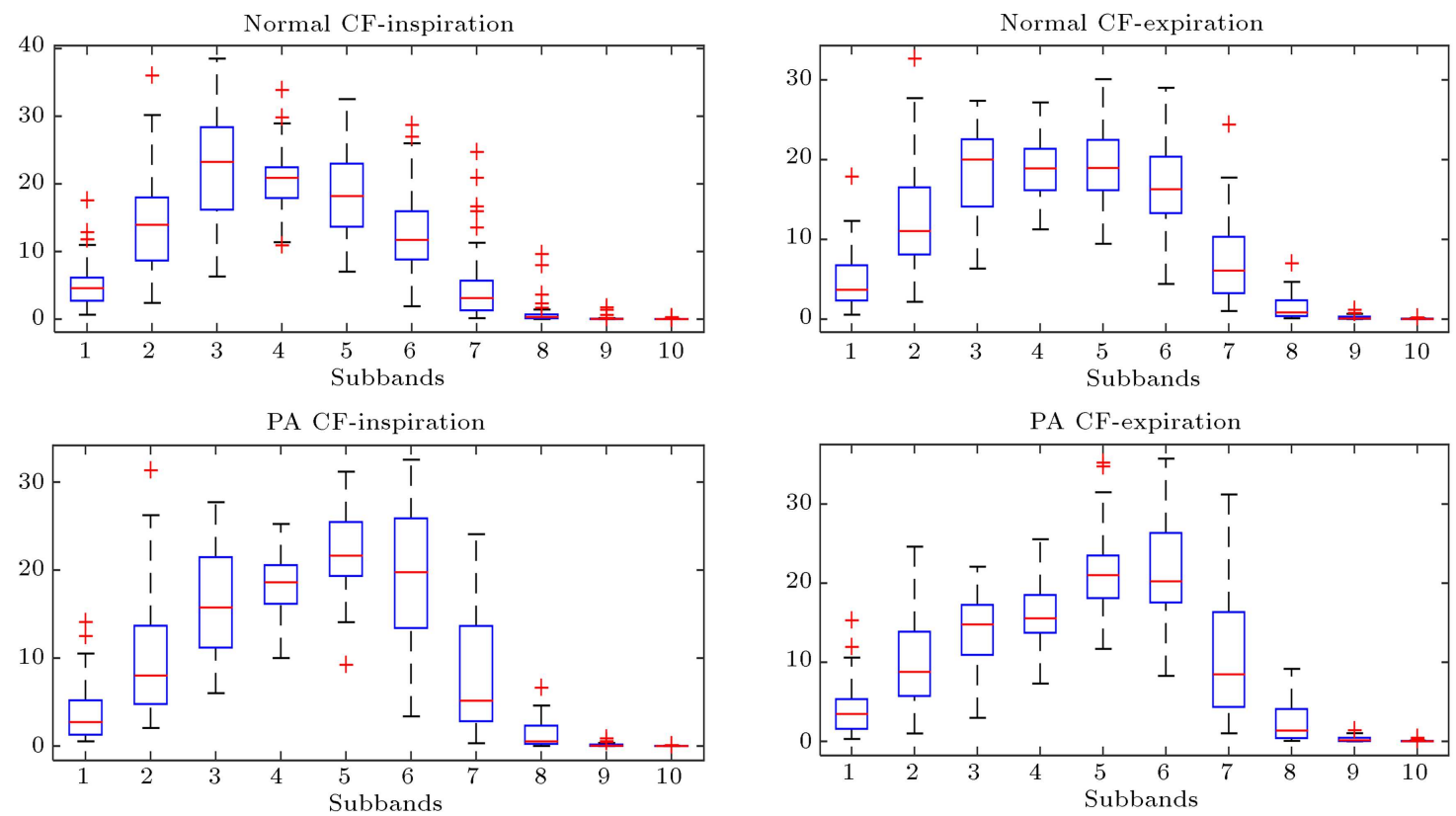

Figure 3. Energy distribution of 10 low Q-factor components of inspiration and expiration sounds in two groups of normal CF and PA CF subjects with median and IQR calculated across all patients.

components of inspiration/expiration sounds $(q=1-$ $-6)$.

As shown in Figure 1, seven feature sets were generated as a single feature vector or a combination of different feature vectors as follows:
1. High Q-factor features extracted from inspiration sound signals (HQ-insp);

2. High Q-factor features extracted from expiration sound signals (HQ-exp); 
3. Low Q-factor features extracted from inspiration sound signals (LQ-insp);

4. Low Q-factor features extracted from expiration sound signals (LQ-exp);

5. High Q-factor and Low Q-factor features extracted from inspiration sound signals (HQ-insp and LQinsp);

6. High Q-factor and Low Q-factor features extracted from expiration sound signals (HQ-exp and LQ$\exp )$;

7. High Q-factor and low Q-factor features extracted from inspiration and expiration sound signals together (HQ-insp and LQ- insp and HQ-exp and LQ$\exp )$.

\subsection{Feature selection}

In order to reduce the number of features, a feature selection algorithm was implemented based on Genetic Algorithm (GA). GA selected the best group of features by searching for possible feature combinations. It is a kind of evolutionary algorithms [32] and basic steps of the implemented algorithm are described as follows:

- Generating random initial population of chromosomes. The chromosome length was considered equal to the total number of features. Chromosome bits denote whether or not the features were selected by assigning 1 or 0 to them, respectively;

- Computing fitness for each chromosome using a defined cost function. GA cost function was proposed based on the classifier results. It also contained a term to control the number of features. More details are represented in Eq. (16) in the results section;

- Selecting the best chromosomes based on the fitness selection (a larger fitness value corresponds to a better or lower cost) can be done using different methods such as stochastic uniform selection, Roulette wheel selection, etc., among which the latter is used in this study;

- Applying crossover (single point, two points, and uniform crossovers) to chromosomes, of which twopoint crossover was used here. In this method, parent chromosomes are split into three fragments using two selected random points. Then, produced fragments are recombined to create new chromosomes;

- Applying mutation operators to individuals. Random bits of chromosomes were selected in the mutation. Then, new chromosomes were created by inverting selected bits (1 changed into 0 , and vice versa);

- Keeping the best individual that was found for the next generation;
- Repeating crossover, mutation, and selection until a stopping condition is met.

By implementing the GA algorithm, the best collection of the features was selected from a subset of possible combinations of features in a feature set.

\subsection{Classification}

After selecting appropriate features from each feature set, the final feature vectors were normalized in the range of $[0,1]$ to make an efficient classification. Then, normalized feature vectors were fed to classifiers. At first, an SVM classifier was examined to discriminate PA CF patients' respiratory sounds from Normal CF ones. Then, an ensemble decision was proposed based on the majority voting of the class labels given by three SVM classifiers applied to three feature groups. Features were fed to classifiers by applying 'leave-oneout' cross-validation method to subjects, meaning that the classifier was trained on all features except those of one subject. Then, the remaining features were used for test to determine the existence of PA infection based only on one respiratory cycle. This step was repeated for all the other subjects.

\subsubsection{Support Vector Machine (SVM)}

SVM finds an optimal hyperplane between samples of two classes that are linearly separable (or can be separated linearly by moving to another space using a kernel function) [33]. Assume that $x_{d}$ is the $d$ th sample and $y_{d}$ is its label ( $d$ is the index of samples); SVM tries to minimize $L(\mathrm{Eq}$. (16)) with respect to $\vec{w}$ and $b$, where $a_{d} \geq 0, d=1, \ldots, K_{s}$ are Lagrange coefficients:

$$
L=\frac{1}{2} \vec{w}-\sum_{d=1}^{K_{s}} a_{d} y_{d}\left(\vec{w} \vec{x}_{d}+b\right)+\sum_{d=1}^{K_{s}} a_{d} .
$$

By computing $\vec{w}$ and $b$, optimum hyperplane will be obtained [34].

In this study, a nonlinear SVM classifier with radial basis (Gaussian) function kernel with radius 1 was used. Sequential Minimal Optimization (SMO) was employed to find the separating hyper-plane.

\subsubsection{Ensemble classification}

An ensemble classifier combines a number of simple classifiers to improve the performance of classification. This combination has been addressed by different names in the literature, including classifier fusion, the mixture of experts, dynamic classifier selection, divide-and-conquer classifiers, etc. [35]. The main reason for using Ensemble learning is to improve the generalization ability of classifier decisions.

In this study, results of applying three SVM classifiers to three feature groups were combined to determine the existence of PA infection in CF patients. Majority voting was used as a combination method, in 
which if more than one classifier identifies a subject as $\mathrm{PA} \mathrm{CF}$ or normal $\mathrm{CF}$, the result will be categorized in that group.

\section{Results}

The proposed method was applied to the recorded lung sounds of CF patients with the purpose of finding appropriate feature sets to diagnose CF patients with PA infection. Seven feature sets were created and the best group of features was selected by applying GA to each feature set. GA parameters were selected as Roulette Wheel selection function, uniform mutation function, two-point crossover function, and a generation number of 500. Cost function $E$ was defined as Eq. (17):

$$
E=\sqrt{\left(1-A c c_{1}\right)^{2}+\left(1-A c c_{2}\right)^{2}}+0.001 \times N_{f}
$$

where $A c c_{1}$ and $A c c_{2}$ are the sensitivity and specificity of PA infection detection, respectively, after applying the SVM to a group of features and $N_{f}$ is the number of features in that group. Some feature extraction methods assign a rank number to each feature. In those methods, a special number of features (a fix number) are selected based on their ranking from the best to the worst rank. In comparison with those methods, GA can automatically select an appropriate number of features. The constraint term in Eq. (17) controls the number of selected features; this means that when two feature sets result in the same classification error, the one with the smaller number of features is given a lower $E$ value. Some trial-and-error tests were performed to configure the GA for limiting the number of features. Then, a value of 0.001 was selected as the coefficient of the second term in Eq. (17).

GA was implemented 30 times by assigning different values to crossover probability $(0.6-0.8)$ and mutation probability $(0.05-0.1)$ and creating a different random initial population.

Figure 4 shows the percentage of selected features from each feature set no. 1 to 4 (HQ-insp, HQ-exp, LQ-insp and LQ-exp) in the feature set 5-7. GA converged to the same kind of results using different initial populations (different or equal number of $L Q$ and HQ features). It can be seen in the two top pies that

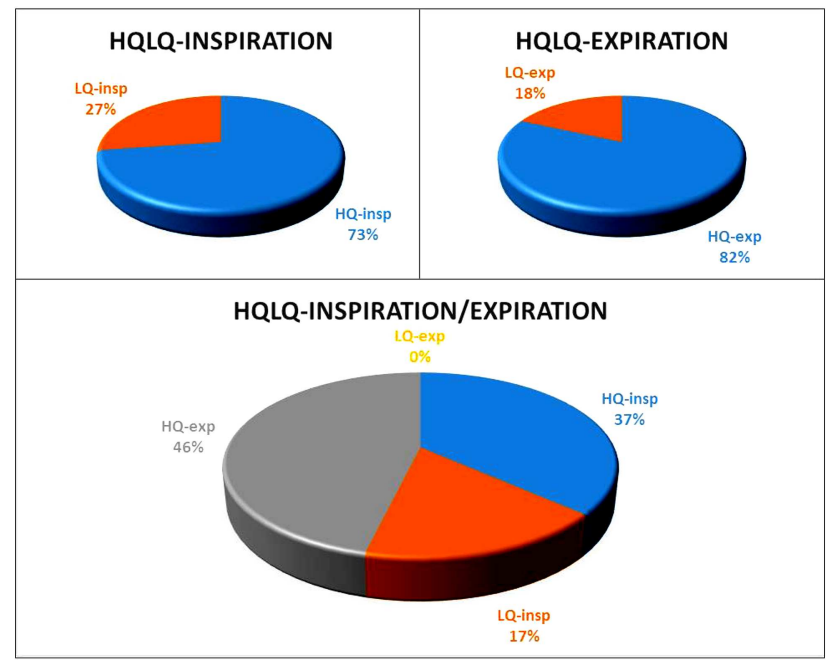

Figure 4. The percentage of selected features from the feature sets 1-4 (HQ-insp, HQ-exp, LQ-insp, and LQ-exp) in the feature sets $5-7$.

the percentage of selected HQ features is higher than that of LQ features in both inspiration and expiration sounds. Therefore, they may be more effective than LQ features in the detection of PA infection. The button pie shows that HQ features are superior to LQ features, and HQ-expiration features are superior to HQ-inspiration features.

As mentioned before, GA feature selection was binary; hence, the length of the selected features can be different in the initial population, which may also change by crossover and mutation operators. Therefore, the length of the selected features could be different in each of the $30 \mathrm{GA}$ implementations. However, GA feature selection usually converges to some specific feature groups. The means and standard deviations of selected feature lengths and accuracies for the seven proposed feature sets are compared in Table 2. As shown in Table 2, feature set 7 , which is a combination of all features, results in the best average accuracy by a value of $82.0 \pm 3.2 \%$.

In comparison between HQ-insp and HQ-exp features, HQ-exp features provide better average accuracy. As expected from Figure 4, LQ-insp and LQ-exp features (feature sets 3 and 4) provide lower average accuracy than HQ-insp and HQ-exp. However, LQ

Table 2. Mean and standard deviation of feature lengths and (train/test) accuracies for selected features after applying GA.

\begin{tabular}{lccccccc}
\hline & HQ-insp & HQ-exp & LQ-insp & LQ-exp & $\begin{array}{c}\text { HQ-insp \& HQ-exp \& } \\
\text { LQ- insp }\end{array}$ & $\begin{array}{c}\text { HQ-insp \& } \\
\text { LQ-exp } \\
\text { HQ-exp \& LQ-exp }\end{array}$ \\
\hline $\begin{array}{l}\text { No. of features } \\
\text { Accuracy }\end{array}$ & $5.05 \pm 0.7$ & $4.2 \pm 1.1$ & $4.7 \pm 0.6$ & $3.2 \pm 0.6$ & $4.4 \pm 1.1$ & $5.2 \pm 1.1$ & $4.6 \pm 0.9$ \\
$\begin{array}{l}\text { (train) } \\
(\text { test })\end{array}$ & $(87.2 \pm 2.5)$ & $(84.6 \pm 2.5)$ & $(76.8 \pm 1.9)$ & $(76.9 \pm 3.6)$ & $(84.4 \pm 2.5)$ & $(87.8 \pm 4.3)$ & $(\mathbf{8 9 . 3} \pm \mathbf{3 . 2})$ \\
\hline
\end{tabular}


features (feature sets 3 and 4) provide lower standard deviation than HQ features (feature sets 1 and 2). The smaller number of features in LQ feature sets than HQ feature sets results in less variation in selected features, which can be the reason for the lower standard deviation of the accuracy of LQ features. The average accuracy of feature set 6 (HQ-exp \& LQ-exp) is higher than that of feature set 5 (HQ-insp \& LQ-insp). Features that are extracted from expiration sound signals provide greater accuracy than inspiration sound signals for HQ features, while this is inverse for LQ features.

The best selected features in the feature sets 17 are shown in Table 3 . The proposed method was applied to respiratory sounds of left and right lungs separately, which yielded similar results.

As can be seen, the existence of PA infection in CF patients can be detected based on only one cycle of their respiratory sound samples recorded from the right or left side. Therefore, both sound samples were used together to increase the number of samples. Sensitivity, specificity, and accuracy of detecting PA infection for all the sound samples are reported in percentage in Table 4.

As shown in Table 4, the best result for the detection of infection is obtained from the feature set 7 by sensitivity, specificity, and accuracy of $88.0 \%$,
$87.2 \%$, and $87.7 \%$, respectively. The second best accuracy is obtained from the feature set 6 .

PA infection detection is vital to early initiation of eradication therapy and also, it is essential to reduce the wrong detection to prevent antibiotic overuse. To increase sensitivity (PA infection detection) and specificity (reduce the wrong detection), an ensemble classification was applied to feature sets 1-7. The proposed ensemble classifier was a combination of three SVM classifiers applied to the best three selected feature groups of each feature set. Table 5 presents the parameters of sensitivity, specificity, and accuracy after applying the ensemble classifier to seven feature sets. Besides, the ensemble classifier was applied to three selected feature groups of different feature sets (feature sets 1, 6, 7; feature sets 2, 6, 7; and feature sets $5,6,7)$.

Table 5 shows that combining the three SVM increases accuracies in some cases. The increased accuracies after applying the ensemble classifier are shown in bold in the last column of Table 5. Errors of the three SVM classifiers are correlated in other cases, and the ensemble classifier does not change efficiently by combining these classifiers. The highest accuracy is obtained from a combination of feature sets $5,6,7$ and feature sets $1,6,7$ by accuracy of $90.3 \%$. The

Table 3. Performance results of the best selected features for the feature sets 1-7 for respiratory sounds of left and right lungs.

\begin{tabular}{llccc}
\hline & \multicolumn{1}{c}{ Feature set } & SVM (left+right) & SVM (right) & SVM (left) \\
\hline & & $\begin{array}{c}\text { Acc. } \\
\text { (train/test) }\end{array}$ & $\begin{array}{c}\text { Acc. } \\
\text { (train/test) }\end{array}$ & $\begin{array}{c}\text { Acc. } \\
\text { (train/test) }\end{array}$ \\
\hline $\mathbf{1}$ & HQ-insp & $(87.7 / 79.8)$ & $(87.7 / 80.4)$ & $(87.8 / 79.4)$ \\
$\mathbf{2}$ & HQ-exp & $(86.7 / 78.9)$ & $(85.9 / 76.1)$ & $(85.7 / 79.4)$ \\
$\mathbf{3}$ & LQ-insp & $(77.8 / 72.8)$ & $(78.2 / 78.6)$ & $(77.7 / 66.2)$ \\
$\mathbf{4}$ & LQ-exp & $(75.9 / 71.0)$ & $(74.7 / 65.3)$ & $(74.4 / 69.1)$ \\
$\mathbf{5}$ & HQ-insp \& LQ- & $(82.3 / 78.9)$ & $(82.2 / 84.7)$ & $(82.6 / 73.5)$ \\
$\mathbf{6}$ & HQ-exp \& LQ-exp & $(98.1 / 81.5)$ & $(98.2 / 80.4)$ & $(98.2 / 85.3)$ \\
$\mathbf{7}$ & HQ-insp \& LQ insp and HQ-exp \& LQ-exp & $(\mathbf{9 2 . 1 / 8 7 . 7 )}$ & $(92.2 / 84.7)$ & $(92.7 / 89.3)$ \\
\hline
\end{tabular}

Table 4. Performance results of the best selected features for the feature sets $1-7$ in percentage.

\begin{tabular}{lcccc}
\hline & & \multicolumn{3}{c}{ SVM } \\
\cline { 3 - 4 } & Feature set & $\begin{array}{c}\text { Spec. N } \\
\text { (train/test) }\end{array}$ & $\begin{array}{c}\text { Sens. PA } \\
\text { (train/test) }\end{array}$ & $\begin{array}{c}\text { Acc. } \\
\text { (train/test) }\end{array}$ \\
\hline 1 & HQ-insp & $(76.7 / 65.9)$ & $(95.4 / 89.5)$ & $(87.7 / 79.8)$ \\
2 & HQ-exp & $(82.9 / 74.4)$ & $(89.4 / 82.0)$ & $(86.7 / 78.9)$ \\
3 & LQ-insp & $(72.4 / 70.2)$ & $(81.8 / 74.6)$ & $(77.8 / 72.8)$ \\
4 & LQ-exp & $(72.1 / 61.7)$ & $(78.6 / 77.6)$ & $(75.9 / 71.0)$ \\
5 & HQ-insp \& LQ- insp & $(78.8 / 72.3)$ & $(84.8 / 83.5)$ & $(82.3 / 78.9)$ \\
6 & HQ-exp \& LQ-exp & $(97.8 / 70.2)$ & $(98.4 / 89.5)$ & $(98.1 / 81.5)$ \\
7 & HQ-insp \& LQ insp and HQ-exp \& LQ-exp & $\mathbf{( 9 3 . 6 / 8 7 . 2 )}$ & $\mathbf{( 9 0 . 9 / 8 8 . 0 )}$ & $\mathbf{( 9 2 . 1 / 8 7 . 7 )}$ \\
\hline
\end{tabular}


Table 5. Sensitivity, specificity, and accuracy of applying ensemble classifier to 7 feature sets in percentage.

\begin{tabular}{|c|c|c|c|c|c|c|c|c|}
\hline \multicolumn{2}{|r|}{ Feature set } & & \multirow{2}{*}{$\frac{\text { SVM1 }}{\text { Acc. }}$} & \multirow{2}{*}{$\frac{\text { SVM2 }}{\text { Acc. }}$} & \multirow{2}{*}{$\begin{array}{c}\text { SVM3 } \\
\text { Acc. }\end{array}$} & \multicolumn{3}{|c|}{ Ensemble classifier } \\
\hline & & & & & & Spec. N & Sens. PA & Acc. \\
\hline \multirow{2}{*}{\multicolumn{2}{|c|}{1 HQ-insp }} & Train & 87.7 & 86.2 & 86.5 & 84.9 & 92.9 & 89.4 \\
\hline & & Test & 79.8 & 77.1 & 77.1 & 74.4 & 86.5 & 81.5 \\
\hline \multirow{2}{*}{\multicolumn{2}{|c|}{2 HQ-exp }} & Train & 86.7 & 85.7 & 83.7 & 87.3 & 90.2 & 88.6 \\
\hline & & Test & 78.9 & 78.9 & 78.9 & 80.8 & 82.0 & 81.5 \\
\hline \multirow[t]{2}{*}{3} & LQ-insp & Train & 77.8 & 76.3 & 76.3 & 75.9 & 76.6 & 76.3 \\
\hline & & Test & 72.8 & 72.8 & 72.8 & 70.2 & 74.6 & 72.8 \\
\hline \multirow[t]{2}{*}{4} & LQ-exp & Train & 75.9 & 75.9 & 75.2 & 74.7 & 76.7 & 75.9 \\
\hline & & Test & 71.0 & 70.1 & 70.1 & 70.1 & 70.2 & 70.1 \\
\hline \multirow[t]{2}{*}{5} & HQ-insp \& LQ- insp & Train & 82.3 & 82.3 & 89.3 & 78.6 & 85.1 & 82.4 \\
\hline & & Test & 78.9 & 78.9 & 72.8 & 72.3 & 83.5 & 78.9 \\
\hline \multirow[t]{2}{*}{6} & HQ-exp \& LQ-exp & Train & 98.1 & 94.6 & 88.7 & 97.8 & 99.8 & 99.1 \\
\hline & & Test & 81.5 & 80.7 & 79.8 & 76.5 & 89.5 & 84.2 \\
\hline \multirow[t]{2}{*}{7} & HQ-insp \& LQ- insp and HQ-exp \& LQ-exp & Train & 92.1 & 82.4 & 98.1 & 93.5 & 98.1 & 96.5 \\
\hline & & Test & 87.7 & 86.8 & 85.9 & 87.2 & 88.0 & 87.7 \\
\hline \multirow[t]{2}{*}{8} & Feature sets $1,6,7$ & Train & 87.7 & 98.1 & 92.1 & 96.7 & 98.7 & 97.9 \\
\hline & & Test & 79.8 & 81.5 & 87.7 & 82.9 & 95.5 & 90.3 \\
\hline \multirow[t]{2}{*}{9} & Feature sets $2,6,7$ & Train & 86.7 & 98.1 & 92.1 & 96.7 & 97.8 & 97.4 \\
\hline & & Test & 78.9 & 81.5 & 87.7 & 78.7 & 94.0 & 87.7 \\
\hline & Feature sets $5,6,7$ & Train & 82.3 & 98.1 & 92.1 & 96.7 & 95.5 & 95.9 \\
\hline & & Test & 78.9 & 81.5 & 87.7 & 85.1 & 94.0 & 90.3 \\
\hline
\end{tabular}

Table 6. The probability of being classified as PA CF for features of PA CF patients before and after treatment.

\begin{tabular}{lccc}
\hline Feature set & $\begin{array}{c}\text { Before } \\
\text { treatment } \\
\text { (mean } \pm \text { std })\end{array}$ & $\begin{array}{c}\text { One month } \\
\text { after treatment }\end{array}$ & P-value \\
(mean \pm std $)$ & \\
\hline HQ-insp \& LQ- insp and HQ-exp, \& LQ-exp & $0.75 \pm 0.20$ & $0.62 \pm 0.24$ & 0.003 \\
Feature sets 1, 6,7 & $0.87 \pm 0.09$ & $0.55 \pm 0.09$ & $<0.001$ \\
Feature sets 2, 6,7 & $0.81 \pm 0.11$ & $0.64 \pm 0.09$ & $<0.001$ \\
Feature sets 5, 6,7 & $0.84 \pm 0.09$ & $0.57 \pm 0.11$ & $<0.001$ \\
\hline
\end{tabular}

two mentioned ensemble classifiers (items 8 and 10 in Table 5) result in sensitivity rates of $95.5 \%$ and $94.0 \%$ and specificity rates of $82.9 \%$ and $85.1 \%$, respectively. Results show that TQWT features are capable of diagnosing respiratory cycles with PA infection.

To demonstrate the effectiveness of the proposed method, respiratory sounds of PA CF patients, which were recorded one month after treatment, were investigated. For these respiratory sounds, the probability of being classified as PA CF was computed by the best selected feature groups in Table 5. These probabilities were compared with that of classifying PA CF patients into PA CF class before treatment. To this end, after training SVM, the posterior probability of assigning a sample to a class was obtained using the method in
[34]. After that, the existence of significant differences was investigated between the probabilities of these two groups (CF patients before and after the treatment of PA infection) using a two-sample t-test with a $P$-value of 0.05. The probabilities for assigning respiratory sounds to PA CF class before and after treatment are shown in Table 6 for the best ensemble classifiers of Table 5 .

As can be seen in Table 6, the probability of identifying respiratory sounds of PA CF subjects as PA infection after the treatment decreased significantly by the proposed method.

Feature/parameter selection and training steps were done using noise-free signals. The performance of the proposed method was investigated in the presence 
of noises and artifacts using noisy respiratory sound signals. Three noisy conditions were examined as follows:

- Raw noisy signals: respiratory sound signals after discarding speaking and coughing segments without applying any additional noise removal technique (raw noisy signals contaminated with digital stethoscope movement noises and cardiac sounds);

- Digital stethoscope movement noises: Raw noisy signals after cardiac sound effect removal;

- Cardiac sound effect: Raw noisy signals after removing digital stethoscope movement noises.

The best features in Table 5 were extracted from these three noisy signals. Then, the accuracy of PA infection detection was computed. The results are shown in Table 7 .

As shown in Table 7, the presence of digital stethoscope movement noises and cardiac sounds reduced the accuracy by about $10 \%$ in comparison with noise-removed signals. In noisy conditions, the accuracy was reduced no more than $14 \%$. The results show that the proposed features are appropriate for this study and that the performance of the method is satisfactory in the presence of noise.

The following are the final obtained results:

- High Q-factor features are superior to low Q-factor features in terms of PA detection accuracy since the former are selected more than the latter in feature sets, which contain both types of features and result in better accuracies;

- Based on the comparison between inspiration and expiration sound features, none of the two is superior to the other in terms of PA detection accuracy. Accuracies for HQ expiration features are better than those for HQ inspiration features and they are reversed for LQ features;

- The best groups of features represent a combination of high Q-factor and low Q-factor features in inspiration and expiration (feature set 7 );

- Using ensemble decision increased the average accuracy of detecting $\mathrm{PA}$ infection. The best result of detecting PA infection is obtained from combining the three best feature sets by the ensemble classifier. Results indicate that normal $\mathrm{CF}$ and $\mathrm{PA} \mathrm{CF}$ patients' respiratory sound signals are distinguishable with an accuracy of $90.3 \%$;

- The probability of classifying respiratory sounds as PA CF is reduced significantly after the treatment of PA CF subjects by the proposed groups of features;

- Results show that the performance of the proposed method is satisfactory in the presence of noises and artifacts.

\section{Discussion}

As mentioned above, the diagnosis of PA infection in the primary stage is essential to initiate the treatment and reduce the risk of chronic infection. The gold standard method for detecting PA infection is time consuming. Therefore, a rapid diagnostic method can facilitate eradication therapy. For the first time, this research has examined the capability of respiratory sound to diagnose PA infection in $\mathrm{CF}$ patients.

Although there are some studies on the topic of the relationship between increasing sputum and respiratory sound samples (as summarized in Table 8 ), there has been no research on the detection of PA infection in CF patients using respiratory sounds.

As shown in Table 8, 12 frequency features were used in [28] to discriminate sputum from non-sputum respiratory sounds with an accuracy rate of $85 \%$ $97 \%$. In [25], 16 features of respiratory sounds in the time-frequency domain and 14 features in [27] derived from discrete wavelet transform of respiratory sounds achieved accuracies of $83.5 \%$ and $84.53 \%$ in discriminating sputum from non-sputum states, respectively. In another study, 46 features derived from the EMD method achieved an accuracy rate of $92.02 \%$ in classifying sputum from non-sputum states. In this study, TQWT analysis was used. The results of TQWT analysis are consistent with previous studies that revealed the effectiveness of time-frequency features in detecting sputum increase. In comparison with other investigations [25-28], the proposed method achieved an acceptable accuracy (90.3\%) with the

Table 7. Results of the proposed method in the presence of noises and artifacts.

\begin{tabular}{|c|c|c|c|c|c|c|c|c|c|c|c|c|}
\hline & \multicolumn{3}{|c|}{ Without noise } & \multicolumn{3}{|c|}{ Raw noisy signal } & \multicolumn{3}{|c|}{$\begin{array}{c}\text { Digital } \\
\text { stethoscope } \\
\text { movement noises }\end{array}$} & \multicolumn{3}{|c|}{$\begin{array}{c}\text { Cardiac sound } \\
\text { effect }\end{array}$} \\
\hline & $\begin{array}{l}\text { Spec. } \\
\mathrm{N}\end{array}$ & $\begin{array}{l}\text { Spec. } \\
\mathrm{N}\end{array}$ & Acc. & $\begin{array}{l}\text { Spec. } \\
\mathrm{N}\end{array}$ & $\begin{array}{l}\text { Spec. } \\
\text { N }\end{array}$ & Acc. & $\begin{array}{c}\text { Spec. } \\
\mathrm{N}\end{array}$ & $\begin{array}{c}\text { Sens. } \\
\text { PA }\end{array}$ & Acc. & $\begin{array}{c}\text { Spec. } \\
\mathbf{N}\end{array}$ & $\begin{array}{c}\text { Sens. } \\
\text { PA }\end{array}$ & Acc. \\
\hline Feature sets $1,6,7$ & 82.9 & 70.2 & 90.3 & 68.1 & 82.1 & 76.3 & 70.2 & 86.5 & 79.8 & 72.3 & 88.0 & 81.5 \\
\hline Feature sets $5,6,7$ & 85.1 & 74.4 & 90.3 & 63.8 & 88.0 & 78.1 & 74.4 & 85.1 & 80.7 & 74.4 & 86.5 & 81.5 \\
\hline
\end{tabular}


Table 8. Some related studies investigating respiratory sounds to diagnose sputum condition.

\begin{tabular}{|c|c|c|c|c|}
\hline Study & Number of participants & Features & \# Features & Accuracy \\
\hline $\begin{array}{l}\text { TQWT features } \\
\text { (Proposed method) }\end{array}$ & $\begin{array}{l}25 \text { patients, } 114 \text { respiratory sound } \\
\text { cycles and } 11 \text { patients after treatment } \\
\text { (22 respiratory sound cycles) }\end{array}$ & $\begin{array}{l}\text { Features from TQWT analysis of } \\
\text { respiratory sound signals }\end{array}$ & 15 & $90.2 \%$ \\
\hline $\begin{array}{l}\text { Center of gravity } \\
\text { features }[28]\end{array}$ & $\begin{array}{l}3 \text { patients and } 128 \text { respiratory } \\
\text { sound samples }\end{array}$ & $\begin{array}{l}\text { Center of gravity in a } \\
\text { frequency domain }\end{array}$ & 12 & $85 \%-97 \%$ \\
\hline $\begin{array}{l}\text { Time-frequency image } \\
\text { features [25] }\end{array}$ & $\begin{array}{l}12 \text { patients and } 272 \text { respiratory } \\
\text { sound samples }\end{array}$ & $\begin{array}{l}\text { Features from time frequency } \\
\text { distribution of respiratory sound } \\
\text { signals }\end{array}$ & 16 & $83.5 \%$ \\
\hline $\begin{array}{l}\text { Discrete wavelet } \\
\text { transform features [27] }\end{array}$ & 595 sound samples & $\begin{array}{l}\text { Features extracted from } \\
\text { discrete wavelet transform }\end{array}$ & 14 & $84.53 \%$ \\
\hline EMD features [26] & 14 patients and 803 sound samples & $\begin{array}{l}\text { Features based upon Empirical } \\
\text { mode decomposition }\end{array}$ & 46 & $92.02 \%$ \\
\hline
\end{tabular}

smaller number of features (15 features). Furthermore, the proposed method was examined after the infection treatment and it was effective in the investigation of PA infection treatment. Two methods (EMD features and center of gravity in frequency domain features), which had better accuracies than the other methods, were selected from Table 8 . These features were extracted from recorded respiratory sounds of CF patients. The numbers of EMD features and center of gravity features are 46 and 12. GA feature selection was applied to these two feature sets to select the best features of each feature set. Results of these two methods were compared with that of the proposed method. TQWT features resulted in better accuracies (7th row in Table 4: sensitivity: $87.2 \%$, specificity: $88.0 \%$, and accuracy: $87.7 \%$ ) than EMD (sensitivity: $79.1 \%$, specificity: $72.3 \%$, and accuracy: $76.3 \%$ ) and center of gravity features (sensitivity: $55.2 \%$, specificity: $70.2 \%$, and accuracy: $61.4 \%$ ). Results show that differences between spectral properties of lung sounds in PA CF and normal CF are emphasized when the pulmonary sounds are decomposed with TQWT filters. This effect may result from better localization of small vibrations caused by increasing sputum.

Since infection increases mucus and thickens airways, it was expected that high Q-factor components could result in better accuracies than low Q-factor components in differentiating between Normal CF and PA CF respiratory sound cycles. This is due to their higher resolution than low Q-factor. Consistently, results demonstrated the expectation and the best accuracies were obtained from features of high Q-factor components. Features extracted from both high Qfactor components of inspiration and expiration sounds achieved the best accuracy. Moreover, the ensemble classifier increased the accuracy.
The results of this study demonstrated that the respiratory sounds of $\mathrm{CF}$ patients were affected by PA infection. Furthermore, the proposed features, extracted from the TQWT analysis of respiratory sounds, could detect the changes. Although the proposed approach was able to detect PA infection in CF patients, the present study was limited by its small number of participants. Clinical use of this method needs further research with a larger number of patients involving other infections in CF patients. The aim of this research was to find whether PA infection had an effect on lung sounds of CF patients which could be identified based on the lung sound analysis. Recorded respiratory sound samples had good quality. Given the presence of some noises in the recorded sound signals and noise removal in the preprocessing step, the effects of different noises were investigated in this study. Results show that the proposed method can detect PA infection, even in the presence of noise. In the future, results of this project will be used to design an automatic system to detect PA infection in CF patients for the clinical use. Therefore, all the preprocessing steps for respiratory sound preparation will be implemented as a package to be used for all respiratory sound signals, making this work fully reproducible.

In future work, the proposed method can be used for monitoring the treatment in CF patients by recording respiratory sounds in different time intervals after initiating the treatment. Subsequently, physicians can follow up a patient's status using the proposed approach. As another work, the effect of other infections such as Staphylococcus aureus can be investigated on respiratory sound. Additionally, the results of the proposed method of detecting PA infection were only validated for $\mathrm{CF}$ patients. One suggestion is to apply 
the proposed method to respiratory sound signals of other lung diseases such as other lung infections in nonCF patients. Structural airway alterations in obstructive disease (such as chronic obstructive pulmonary disease) may be different from increasing mucus and sputum in CF patients. Different structural changes in airways cause different changes in respiratory sounds. Therefore, the effectiveness of the proposed features can be investigated in the detection of these changes and discrimination of respiratory disease with obstructive airway structures from those related to mucus airway structures.

\section{Conclusion}

This study proposed an innovative method to detect PA infection in respiratory sounds. For the first time, some lung sound features were introduced for the detection of Pseudomonas Aeruginosa (PA) infection in Cystic Fibrosis (CF) patients. The proposed TQWTrelated features successfully discriminated respiratory sound signals of PA CF patients from normal CF ones. The findings also revealed that the probability of classifying respiratory sound signals of CF patients as PA CF was reduced after the treatment of PA infection. The proposed method utilized lung sound, which is a fast, low-priced, and accessible procedure. Furthermore, it can be helpful in deciding on both preventive and therapeutic strategies.

\section{Acknowledgments}

We thank the team of physicians, pharmacologists, and microbiologists for collaboration to collect data in Pediatric Respiratory and Sleep Medicine Research Center of Children's Medical Center.

\section{Funding}

This research did not receive any specific grant from funding agencies in the public, commercial, or not-for-profit

\section{References}

1. Pillarisetti, N., Williamson, E., Linnane, B., et al. "Infection, inflammation, and lung function decline in infants with cystic fibrosis", American Journal of Respiratory and Critical Care Medicine, 184(1), pp. 75-81 (2011).

2. Ahlgren, H.G., Benedetti, A., Landry, J.S., et al. "Clinical outcomes associated with Staphylococcus aureus and Pseudomonas aeruginosa airway infections in adult cystic fibrosis patients", BMC Pulmonary Medicine, 15(1), p. 67 (2015).
3. Liou, T.G., Adler, F.R., FitzSimmons, S.C., et al. "Predictive 5-year survivorship model of cystic fibrosis", American Journal of Epidemiology, 153(4), pp. 345-352 (2001).

4. Li, Z., Kosorok, M.R., Farrell, P.M., et al. "Longitudinal development of mucoid Pseudomonas aeruginosa infection and lung disease progression in children with cystic fibrosis", Jama, 293(5), pp. 581-588 (2005).

5. Langan, K.M., Kotsimbos, T., and Peleg, A.Y. "Managing Pseudomonas aeruginosa respiratory infections in cystic fibrosis", Current Opinion in Infectious Diseases, 28(6), pp. 547-556 (2015).

6. Mogayzel Jr, P.J., Naureckas, E.T., Robinson, K.A., et al. "Cystic Fibrosis Foundation pulmonary guideline. Pharmacologic approaches to prevention and eradication of initial Pseudomonas aeruginosa infection", Annals of the American Thoracic Society, 11(10), pp. 1640-1650 (2014).

7. Pasterkamp, H., Kraman, S.S., and Wodicka, G.R. "Respiratory sounds: advances beyond the stethoscope", American Journal of Respiratory and Critical Care Medicine, 156(3), pp. 974-987 (1997).

8. Pramono, R.X.A., Bowyer, S., and Rodriguez-Villegas, E. "Automatic adventitious respiratory sound analysis: A systematic review", PloS One, 12(5) (2017).

9. Palaniappan, R., Sundaraj, K., and Sundaraj, S. "A comparative study of the SVM and K-nn machine learning algorithms for the diagnosis of respiratory pathologies using pulmonary acoustic signals", BMC Bioinformatics, 15(1), p. 223 (2014).

10. Fernandez-Granero, M.A., Sanchez-Morillo, D., and Leon-Jimenez, A. "Computerised analysis of telemonitored respiratory sounds for predicting acute exacerbations of COPD", Sensors, 15(10), pp. 26978-26996 (2015).

11. Rocha, V., Melo, C., and Marques, A. "Computerized respiratory sound analysis in people with dementia: a first-step towards diagnosis and monitoring of respiratory conditions", Physiological Measurement, 37(11), p. 2079 (2016).

12. Aras, S., CEnel, M., and Gangal, A. "Investigation of an appropriate feature extraction methods based on single channel lung sounds", in 25th Signal Processing and Communications Applications Conference (SIU), pp. 1-4 (2017).

13. Hadjileontiadis, L.J. and Moussavi, Z.M. "Current techniques for breath sound analysis", in Breath Sounds, Springer, pp. 139-177 (2018).

14. Pancaldi, F., Sebastiani, M., Cassone, G., et al. "Analysis of pulmonary sounds for the diagnosis of interstitial lung diseases secondary to rheumatoid arthritis", Computers in Biology and Medicine, 96, pp. 91-97 (2018).

15. Ferrari, S., Silva, M., Guarino, M., et al. "Cough sound analysis to identify respiratory infection in pigs", Computers and Electronics in Agriculture, 64(2), pp. 318-325 (2008). 
16. Becker, K., Scheffer, C., Blanckenberg, M., et al. "Analysis of adventitious lung sounds originating from pulmonary tuberculosis", in Engineering in Medicine and Biology Society (EMBC), 2013 35th Annual International Conference of the IEEE, pp. 4334-4337 (2013).

17. Oliveira, A., Pinho, C., Dinis, J., et al. "Automatic wheeze detection and lung function evaluation-A preliminary study", in HEALTHINF, pp. 323-326 (2013).

18. Oliveira, A., Pinho, C., and Marques, A. "Effects of a respiratory physiotherapy session in patients with LRTI: a pre/post-test study", The Clinical Respiratory Journal, 11(6), pp. 703-712 (2015).

19. Tenhunen, M., Rauhala, E., Huupponen, E., et al. "High frequency components of tracheal sound are emphasized during prolonged flow limitation", Physiological Measurement, 30(5), p. 467 (2009).

20. Bokov, P., Mahut, B., Flaud, P., et al. "Wheezing recognition algorithm using recordings of respiratory sounds at the mouth in a pediatric population", Computers in Biology and Medicine, 70, pp. 40-50 (2016).

21. Selesnick, I.W. and Bayram, I. "Oscillatory plus transient signal decomposition using overcomplete rational-dilation wavelet transforms", In Wavelets XIII, p. $74460 \mathrm{~V}$ (2009).

22. Selesnick, I.W. "Wavelet transform with tunable Qfactor", IEEE Transactions on Signal Processing, 59(8), pp. 3560-3575 (2011).

23. Ulukaya, S., Serbes, G., and Kahya, Y.P. "Overcomplete discrete wavelet transform based respiratory sound discrimination with feature and decision level fusion", Biomedical Signal Processing and Control, 38, pp. 322-336 (2017).

24. Huang, W., Sun, H., and Wang, W. "Resonancebased sparse signal decomposition and its application in mechanical fault diagnosis: A review", Sensors, 17(6), p. 1279 (2017).

25. Niu, J., Shi, Y., Cai, M., et al. "Detection of sputum by interpreting the time-frequency distribution of respiratory sound signal using image processing techniques", Bioinformatics, 34(5), pp. 820-827 (2017).

26. Niu, J., Shi, Y., Shen, D., et al. "The identification of sputum situation based on the sound from the respiratory tract", in 2018 IEEE/ASME International Conference on Advanced Intelligent Mechatronics (AIM), pp. 1166-1171 (2018).

27. Shi, Y., Wang, G., Niu, J., et al. "Classification of sputum sounds using artificial neural network and wavelet transform", Int. J. Biol. Sci, 14(8), p. 938 (2018).

28. Yamashita, T., Tamura, S., Hayashi, K., et al. "Sparse representation of audio features for sputum detection from lung sounds", in Pattern Recognition (ICPR), 2012 21st International Conference on, pp. 2005-2008 (2012).

29. Gnitecki, J. and Moussavi, Z.M. "Separating heart sounds from lung sounds", IEEE Engineering in Medicine and Biology Magazine, 26(1), p. 20 (2007).
30. Emmanouilidou, D., McCollum, E.D., Park, D.E., et al. "Computerized lung sound screening for pediatric auscultation in noisy field environments", IEEE Transactions on Biomedical Engineering, 65(7), pp. 15641574 (2017).

31. Selesnick, I. "TQWT toolbox guide", Electrical and Computer Engineering, Polytechnic Institute of New York University. Available online at: http://eeweb.poly.edu/iselesni/TQWT/index.html (2011).

32. Kramer, O. "Genetic algorithm essentials", 679, Springer (2017).

33. Wu, X., Kumar, V., Quinlan, J.R., et al. "Top 10 algorithms in data mining", Knowledge and Information Systems, 14(1), pp. 1-37 (2008).

34. Theodoridis, S. and Koutroumbas, K., Pattern Recognition (2003).

35. Kuncheva, L.I., Bezdek, J.C., and Duin, R.P. "Decision templates for multiple classifier fusion: an experimental comparison", Pattern Recognition, 34(2), pp. 299-314 (2001).

\section{Biographies}

Arezoo Karimizadeh received BS and MS degrees in Biomedical Engineering at University of Isfahan in 2010 and 2012, respectively. She is currently a PhD student at K.N. Toosi university of Technology. Her research interests remain in the fields of medical signal and image processing as well as medical sound processing.

Mansour Vali is an Assistant Professor in Biomedical Engineering group of K.N. Toosi University of Technology. He received his BSc degree in 1997 from Isfahan University of Technology in Electrical Engineering, MSc degree in 2000 from Sharif University of Technology in Bioelectric Engineering, and PhD degree in 2006 from Amirkabir University of Technology in Biomedical Engineering, Tehran, Iran. He was a faculty member of Biomedical Engineering group at Shahed University from 2007 to 2012. On February 2013, he joined K.N. Toosi University of Technology. His main research interests are "sound and speech processing in medical and psychological assessments". He has developed a new course with the same title at the Electrical Engineering Department of K.N. Toosi University for supplementary students. Also, he is working on large data processing in medical application.

Mohammad Reza Modaresi has practiced Pediatric Pulmonology at Children's Medical Center, Pediatric Center of Excellence since 2010. He is the Founder and Current Chief of the Pediatric Pulmonary Division at Children's Medical Center. He is also the Founder of a large and active program in the state for diagnostic and interventional flexible bronchoscopy. It is available 
for the patients of any age and size including neonate, infant, children, and adolescent. He also designed Pediatric Sleep Lab and a modern full-service facility Infant and Pediatric Pulmonary Function Laboratory which performed various lung function tests for infant, preschool, and school-age patients. Dr. Modaresi is the President of Iran Cystic Fibrosis Foundation and is, also, the Director of CF Center at Children's Medical Center, which provides care for over 1000 patients with cystic fibrosis. 